\title{
Roles of elevated 20-HETE in the breakdown of blood brain barrier and the severity of brain edema in experimental traumatic brain injury
}

\author{
LIYAN LU ${ }^{1,2^{*}}$, MINGLIANG WANG ${ }^{2 *}$, FANG YUAN $^{3}$, XIAOER WEI $^{2}$ and WENBIN LI $^{2,4}$ \\ ${ }^{1}$ Department of Radiology, Nanjing First Hospital, Nanjing Medical University, Nanjing, Jiangsu 210006; Departments \\ of ${ }^{2}$ Radiology and ${ }^{3}$ Neurosurgery, Shanghai Jiao Tong University Affiliated Sixth People's Hospital, Shanghai 200233; \\ ${ }^{4}$ Imaging Center, Kashgar Prefecture Second People's Hospital, Kashgar, Xinjiang 844000, P.R. China
}

Received May 14, 2016; Accepted March 30, 2017

DOI: $10.3892 / \mathrm{mmr} .2018 .8780$

\begin{abstract}
Breakdown of the blood brain barrier (BBB) is a secondary injury following traumatic brain injury (TBI) and can lead to the development of brain edema. However, the factors that contribute to the disruption of the BBB and increase the severity of brain edema in TBI remain to be elucidated. 20-hydroxyeicosatetraenoic acid (20-HETE) is a metabolite of arachidonic acid. The inhibition of 20-HETEsynthesis by HET0016 has been suggested as a strategy to decrease brain edema. The present study aimed to investigate whether the elevated production of 20-HETE in cerebral tissue may contribute to $\mathrm{BBB}$ breakdown and increase the severity of brain edema in rats with TBI. BBB permeability was quantified using dynamic contrast-enhanced magnetic resonance imaging and brain edema was measured according to brain water content. Superoxide production in injured tissue was also assessed. Liquid chromatography-mass spectrometry was used to evaluate 20-HETE production in injured tissue. Western blot analysis was used to assess the expression of occludin, zonula occludens (ZO)-1, matrix metalloproteinase (MMP)-9, and proteins of the c-Jun N-terminal kinase (JNK) pathway. A total of 3, 24 and $72 \mathrm{~h}$ following the induction of TBI, 20-HETE levels, BBB permeability and brain edema were identified to be increased, accompanied by an increase in superoxide production. Conversely, superoxide dismutase levels, in addition to the total antioxidative capability were decreased. In addition, the expression of MMP-9 and proteins of the JNK pathway was upregulated, whereas the expression of occludin and ZO-1
\end{abstract}

Correspondence to: Dr Wenbin Li, Department of Radiology, Shanghai Jiao Tong University Affiliated Sixth People's Hospital, 600 Yi Shan Road, Shanghai 200233, P.R. China

E-mail: liwenbin@sjtu.edu.cn

${ }^{*}$ Contributed equally

Key words: 20-hydroxyeicosatetraenoic acid, blood brain barrier, brain edema, traumatic brain injury was observed to be suppressed. These results suggested that 20-HETE may aggravate BBB disruption following TBI, via enhancing the expression of MMP-9 and tight junction proteins. Furthermore, oxidative stress and the JNK signaling pathway may be involved in BBB dysregulation. In conclusion, the results of the present demonstrated that the production of 20-HETE was increased in cerebral tissue following traumatic injury, thus suggesting that it may contribute to the compromise of BBB integrity and the development of brain edema.

\section{Introduction}

Traumatic brain injury (TBI) has been reported to induce the release of arachidonic acid (AA) from cell membranes (1). AA levels have been indicated to be increased by $1,093 \%$ within $48 \mathrm{~h}$ following TBI and to remain elevated for several days (2). Cytochrome P450 (CYP) enzymes, particularly CYP4A isozymes, catalyze the $\omega$-hydroxylation of AA to 20-hydroxyeicosatetraenoic acid (20-HETE) (3). Previous studies have suggested that 20-HETE may participate in various physiological and pathological processes, including the regulation of vascular tone, cerebral blood flow, cellular proliferation and inflammation; 20-HETE has been identified as a potent vasoconstrictor in cerebral vasculature and it has been reported to suppress inflammatory reactions (4-6). However, contradictory results have indicated that 20-HETE increased the vascular production of reactive oxygen species (ROS) and promoted the activation of nuclear factor- $\kappa \mathrm{B}$ in cerebrovascular endothelial cells, and consequently deteriorated cerebral inflammation (7).

TBI consists of two stages of pathophysiological injury: The primary stage, characterized by brain contusion, parenchymal hemorrhage, subarachnoid hemorrhage and diffuse axonal injury; and the secondary stage, characterized by edema, herniation, ischemia and infarction (8). Secondary injury mechanisms have been reported to contribute to ongoing injury, during which ROS production is elevated. ROS have been implicated in the pathology of acute and chronic brain injuries, as they have been demonstrated to disrupt the blood brain barrier (BBB) and increase cerebral vascular permeability, thus leading to the development of brain edema (8). 
Matrix metalloproteinase (MMP)-9 has been reported to serve critical roles during tissue morphogenesis and repair, in cell death, and it has been associated with the prognosis of neurological diseases $(9,10)$. In animal and human TBI studies, MMP-9 has been demonstrated to contribute to the pathophysiology of brain edema, and its overexpression was associated with an increase in BBB permeability through the degradation of tight junction proteins, such as occludin and zonula occludens (ZO)-1 (11-13). MMP-9 has been reported to be activated by ROS, in addition to by the c-Jun N-terminal kinase (JNK) intracellular signaling pathway during BBB breakdown $(13,14)$, whereas JNK inhibition was identified to protect BBB integrity, via suppression of MMP-9 activation (15). Therefore, it may be hypothesized that 20-HETE can increase the production of ROS and, through the activation of the JNK signaling pathway, increase the expression of MMP-9 and promote the degradation of occludin and ZO-1, eventually resulting in BBB dysfunction. The present study investigated the roles of 20-HETE in BBB dysfunction and brain edema development, following the experimental induction of TBI, and explored the molecular mechanisms underlying these events.

\section{Materials and methods}

Animals and materials. Male Sprague-Dawley (SD) rats ( $\mathrm{n}=89$; age, 4-6 weeks; weight, 180-260 g) were obtained from Shanghai SLAC Laboratory Animal Co., Ltd. (Shanghai, China). Rats were housed under $12 / 12 \mathrm{~h} \mathrm{light/dark} \mathrm{cycles} \mathrm{at}$ $22 \pm 1^{\circ} \mathrm{C}$, a relative humidity of $40-70 \%$ and given food and water ad libitum. The present study was approved by the Institutional Animal Care and Use Committee of Shanghai Jiao Tong University School of Biomedical Engineering (Shanghai, China).

Antibodies against MMP-9 (cat. no. ab119906), occludin (cat. no. ab31721) and ZO-1 (cat. no. ab190085) were purchased from Abcam (Cambridge, UK), and antibodies against rat polyclonal anti-phosphorylated (p)-JNK (cat. no. 9252S) and rat polyclonal anti-p-c-Jun antibodies (cat. no. 2315S) were purchased from Cell Signaling Technology, Inc. (Danvers, MA, USA).

Animal model.SD rats were randomly divided into 2 groups: TBI and sham groups ( $\mathrm{n}=5$ rats/group). Rats in the TBI group were anesthetized with ketamine $(75 \mathrm{mg} / \mathrm{kg})$ and xylazine $(10 \mathrm{mg} / \mathrm{kg}$; Shanghai Rantai Biological Company, Shanghai, China), and mounted on a stereotaxic frame (Stoelting Co., Wood Dale, IL, USA). A $15 \mathrm{~mm}$-long midline scalp incision was made and a craniotomy (6 $\mathrm{mm}$ in diameter) was then performed over the central aspect of the right parietal cortex, $2 \mathrm{~mm}$ lateral to the sagittal suture. Care was taken to keep the dura intact following exposure. A controlled cortical injury model of TBI was then established, using an impact device (PinPoint Precision Cortical Impactor PCI 3000; Hatteras Instruments, Inc., Cary, NC, USA) with a $2.5-\mathrm{mm}$ rounded steel impactor tip. A moderate injury was inflicted in the right parietal cortex, using a deformation depth of $2.5 \mathrm{~mm}$, a velocity of $1.5 \mathrm{~m} / \mathrm{sec}$ and a duration time of $85 \mathrm{msec}$, and the incision was closed. Following recovery of $\sim 1 \mathrm{~h}$ from the injury, the rats were returned to their cages and allowed free access to food and water. Rats in the sham group underwent surgery, similar to the TBI rats, however received no injury. Rats were maintained for 3,24 and $72 \mathrm{~h}$, following the induction of TBI; these rats formed the TBI groups ( $\mathrm{n}=5$ rats/group). In addition, rats were excluded from the study if dural integrity was compromised. To obtain rat brains, the foramen magnum was first transected with scissors. The parietal bone was carefully opened through the foramen magnum and parietal hemostatic forceps were inserted in order to break both sides of the parietal bone with oblique insertion scissors. The olfactory bulb on the parietal was also carefully removed, and one side of the optic nerve was cut in order to probe into the base of the skull; the whole brain could then be tilted and removed. Rat brains were fixed in the $4 \%$ paraformaldehyde for $24 \mathrm{~h}$ at $4^{\circ} \mathrm{C}$, then embedded in paraffin and sliced into $10 \mathrm{~mm}$ sections.

BBB permeability. BBB permeability was investigated at 3, 24 and $72 \mathrm{~h}$ following TBI by dynamic contrast-enhanced magnetic resonance imaging (DCE-MRI) (8). Rats underwent MRI using a clinical 3.0 T MRI scanner with an eight-channel head coil (Intera-achieva SMI-2.1; Philips Healthcare, DA Best, The Netherlands). MRI pulse sequences were as follows: T2 weighted (T2W) image, T2 map, post-T1 weighted image and DCE-MRI. The image processing software (Cine Tool; GE Healthcare Life Sciences, Little Chalfont, UK) was used to obtain the quantitative parameter volume transfer constant $\left(\mathrm{K}^{\text {trans }}\right)$. $\mathrm{K}^{\text {trans }}$ was calculated based on a modified two compartment model (Fig. 1A), where all of the contrast media in the tissue were primarily located in the two chambers, namely, the intravascular space and the extravascular space. In this model, $\mathrm{K}^{\text {trans }}$ was calculated by measuring the accumulation of gadolinium-diethylenetriaminepentacetate in the extravascular-extracellular space. $\mathrm{K}^{\text {trans }}$ was calculated by manually outlining the regions of interest (ROIs). A total of 3 ROIs at all time points were manually drawn in a 2-mm single slice T2W scan, as presented in Fig. 1B (obtained at 72 h). ROIs for the sham and TBI groups included the focal lesion and the contralateral brain areas. The hyperintensity areas of $\mathrm{T} 2 \mathrm{~W}$ images were manually outlined (Fig. 1C) following TBI, using the signal intensity difference of $\mathrm{T} 2 \mathrm{~W}$ (the difference threshold was 300) to define the border between the hyperintensity area and healthy-looking tissue. Additional T2 maps (scaling of 0-150 ms) were used to define the border between the hyperintensity area and healthy-looking tissue when the border was ill-defined in $\mathrm{T} 2 \mathrm{~W}$ images. The focal lesion area refers to the outlined hyperintensity area which may include the cortex, hippocampal tissues, etc. This procedure was performed by two radiologists.

Brain edema. Brain edema was assessed via measuring the tissue water content, as previously described (8). Briefly, The SD rats were subjected to deep anesthesia. After the diaphragm was cut off, the chest wall was cut along the anterior axillary line. The heart was exposed after the chest wall was turned up, and a syringe was inserted via the left ventricle in the ascending aorta, the insertion depth is $\sim 1 \mathrm{~mm}$, and then the brain was decapitated. The wet weight of a 3-mm coronal tissue section of the ipsilateral cortex, centered on the impact site, was measured immediately following sacrifice at 3,24, $72 \mathrm{~h}$ and 7 days post-TBI. Tissue samples were then dried in an oven at $100^{\circ} \mathrm{C}$ for $48 \mathrm{~h}$ to obtain the dry weight. Tissue water content was calculated using the following formula: Water content $=[$ (wet weight - dry weight $) /$ wet weight $] \times 100 \%$. 
Oxidative stress indices. The levels of malondialdehyde (MDA; cat. no. A003-1) and superoxide dismutase (SOD; cat. no. A001-3), and the total antioxidative capacity (T-AOC; cat. no. A015) were measured using commercially available kits (Nanjing Jiancheng Bioengineering Institute, Nanjing, China). Briefly, following TBI (3, 24 and $72 \mathrm{~h}$, and 7 days post-TBI), the left-brain hemispheres were removed and homogenized in ice-cold PBS. The brain tissue was poured into the glass homogenate tube, and the remaining 1/3 homogenate medium or saline was also added to the glass homogenate tube. The tube in the left hand was inserted into the homogenate containing the mixture of water containers. The rod in the right hand was vertically inserted into the casing for dozens of times (6-8 $\mathrm{min}$ ) upper and lower to make the tissue homogenate. The samples were centrifuged at 2,000 x $\mathrm{g}$ for $10 \mathrm{~min}$ at $4^{\circ} \mathrm{C}$ and the supernatants were used to measure MDA, SOD and $\mathrm{T}-\mathrm{AOC}$, according to the manufacturer's protocol.

Liquid chromatography-mass spectrometry (LC-MS). SD rats were anesthetized with ketamine $(75 \mathrm{mg} / \mathrm{kg})$ and xylazine $(10 \mathrm{mg} / \mathrm{kg})$. Brain tissue samples from the area of injury were obtained and frozen immediately at $-80^{\circ} \mathrm{C}$. The brain samples were homogenized as mentioned above on ice and $100 \mathrm{~g}$ homogenate were used for proteomic screening. Deuterated 20-HETE-d 6 ( $2 \mu \mathrm{l})$ (SCIEX, Framington, MA, USA) was added to the homogenates as an internal standard. Subsequently, the samples (each sample was the homogenate from the brain of one rat with PBS) were injected into the LC-MS (TripleTOF ${ }^{\circledR}$ 5600+; SCIEX) following evaporation and reconstitution in the mobile phase with buffer A ( $0.1 \%$ formic acid in water) and buffer $\mathrm{B}$ (98\% acetonitrile and $0.1 \%$ formic acid in water). The samples were quantified by LC-MS (curtain gas: 44 Psi; collision gas: $12 \mathrm{Psi}$; ionspray voltage: 5,000 V; nebulizer current: $6 \mathrm{~A}$; temperature: $55^{\circ} \mathrm{C}$; ion source gas1: $90 \mathrm{Psi}$; ion source gas 2: 90 Psi) and the levels of 20-HETE were measured (Mass Frontier Software v5.0; Thermo Fisher Scientific, Inc., Waltham, MA, USA) in each sample.

Western blot analysis. Brains were removed and the 2 hemispheres were separated. The lesion areas were homogenized in a buffer containing Tris $50 \mathrm{mM}(\mathrm{pH}=7.4), \mathrm{NaCl} 150 \mathrm{mM}$, $1 \%$ Triton X-100, EDTA $1 \mathrm{mM}$, and phenylmethylsulfonyl fluoride $2 \mathrm{mM}$. The homogenates were centrifuged at $500 \mathrm{xg}$ for $15 \mathrm{~min}$ at $4^{\circ} \mathrm{C}$, and the supernatants were collected. Protein concentration in the supernatants was determined using a bicinchoninic acid protein assay kit (Pierce; Thermo Fisher Scientific, Inc.), with bovine serum albumin as the standard. Equal amounts of extracted protein samples $(50 \mu \mathrm{g})$ were separated by $12 \%$ SDS-PAGE and transferred onto nitrocellulose membranes, which were then blocked with $5 \%$ nonfat milk for $2 \mathrm{~h}$ at room temperature. Membranes were probed $\left(4^{\circ} \mathrm{C}\right.$ overnight $)$ with primary antibodies against occludin, ZO-1, MMP-9, p-JNK and p-c-Jun (1:400). They were then incubated at $37^{\circ} \mathrm{C}$ for $1 \mathrm{~h}$ with horseradish peroxidase-conjugated secondary antibodies (cat. nos. 140253, 33725, 3007, 6254 and 6093; Jackson ImmunoResearch Laboratories, Inc., West Grove, PA, USA). Protein bands were visualized using an enhanced chemiluminescence detection system (Amersham; GE Healthcare Life Sciences, Little Chalfont, UK). Blots were semi-quantified using Multi Gauge software (ias3000, Fuji,
Tokyo, Japan) and the results were normalized to those of $\beta$-actin (cat. no. ab8226; 1:1,000; Abcam), which was used as the loading control.

Statistical analysis. All analyses were performed with SPSS v17.0 software (SPSS, Inc., Chicago, IL, USA). Normality testing was performed using the Shapiro-Wilk test and equality of variance was assessed using Levene's test. The statistical significance of the differences between groups was assessed using two-way analysis of variance with a Bonferroni post hoc test. Data are expressed as the mean \pm standard deviation of 3 repeated experiments. $\mathrm{P}<0.05$ was considered to indicate a statistically significant difference.

\section{Results}

Alterations in $K^{\text {trans }}$. An immediate and sustained increase in $\mathrm{K}^{\text {trans }}$ post-injury was observed among rats in the TBI group compared with the sham group. As demonstrated in Fig. 2A, $\mathrm{K}^{\text {trans }}\left(\mathrm{min}^{-1}\right)$ was identified to be significantly increased at 3 $\left(0.024 \pm 0.002\right.$ vs. $\left.0.003 \pm 0.001 \mathrm{~min}^{-1}, \mathrm{P}<0.01\right), 24(0.034 \pm 0.004$ vs. $\left.0.003 \pm 0.001 \mathrm{~min}^{-1}, \mathrm{P}<0.01\right)$ and $72 \mathrm{~h}(0.056 \pm 0.003$ vs. $\left.0.003 \pm 0.003 \mathrm{~min}^{-1}, \mathrm{P}<0.05\right)$ following the induction of TBI compared with the sham group. In addition, the increase in $\mathrm{K}^{\text {trans }}$ appeared to be time-dependent, as $\mathrm{K}^{\text {trans }}$ values were significantly different between the various time points within the TBI group $(\mathrm{P}<0.05$; Fig. $2 \mathrm{~A})$. The increase of $\mathrm{K}^{\text {trans }}$ suggests the extent of the disruption of the BBB.

Brain edema. Brain water content was demonstrated to be increased as early as $3 \mathrm{~h}$ following injury compared with the sham group $(79.62 \pm 0.70$ vs. $78.54 \pm 0.21 \%, \mathrm{P}<0.05)$, thus indicating the extent of brain edema. The significant increase in brain water was sustained at $24(81.44 \pm 0.40$ vs. $78.54 \pm 0.21 \%$, $\mathrm{P}<0.05)$ and $72 \mathrm{~h}(83.44 \pm 0.33$ vs. $78.74 \pm 0.21 \%, \mathrm{P}<0.05)$ following the induction of TBI compared with the sham group (Fig. 2B). In addition, brain water contents among rats in the TBI group were identified to be significantly increased 24 and $72 \mathrm{~h}$ post-injury compared with at $3 \mathrm{~h}$ post-injury ( $\mathrm{P}<0.05$ vs. $3 \mathrm{~h}$ post-TBI; Fig. 2B).

Oxidative stress indices. MDA levels in brain tissue samples from the lesion area were demonstrated to be increased at 3 $(6.04 \pm 0.11$ vs. $3.02 \pm 0.13 \mathrm{nmol} / \mathrm{mg}, \mathrm{P}<0.05), 24(7.38 \pm 0.15$ vs. $3.02 \pm 0.20 \mathrm{nmol} / \mathrm{mg}, \mathrm{P}<0.05)$ and $72 \mathrm{~h}(9.32 \pm 0.08$ vs. $3.02 \pm 0.08 \mathrm{nmol} / \mathrm{mg}, \mathrm{P}<0.05)$ post-injury compared with the sham group (Fig. 3A). Notably, MDA levels were identified to be significantly different between the various time points within the TBI group $(\mathrm{P}<0.05$; Fig. $3 \mathrm{~A})$, thus suggesting an increase in ROS production following TBI.

The present results demonstrated that SOD activity in the lesion area was significantly suppressed 3 (62.44 \pm 0.84 vs.

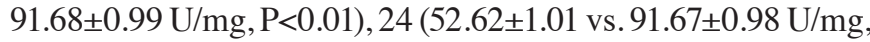
$\mathrm{P}<0.05)$ and $72 \mathrm{~h}(40.43 \pm 0.72$ vs. $91.64 \pm 1.09 \mathrm{U} / \mathrm{mg}, \mathrm{P}<0.05)$ following the induction of TBI compared with the sham group (Fig. 3B). Similarly, the decrease in SOD activity was also identified to be time-dependent within rats in the TBI group $(\mathrm{P}<0.05$; Fig. 3B).

Brain tissue from the lesion area also exhibited reduced T-AOC 3 (6.74 \pm 0.15 vs. $2.14 \pm 0.11 \mathrm{U} / \mathrm{mg}, \mathrm{P}<0.05), 24$ 

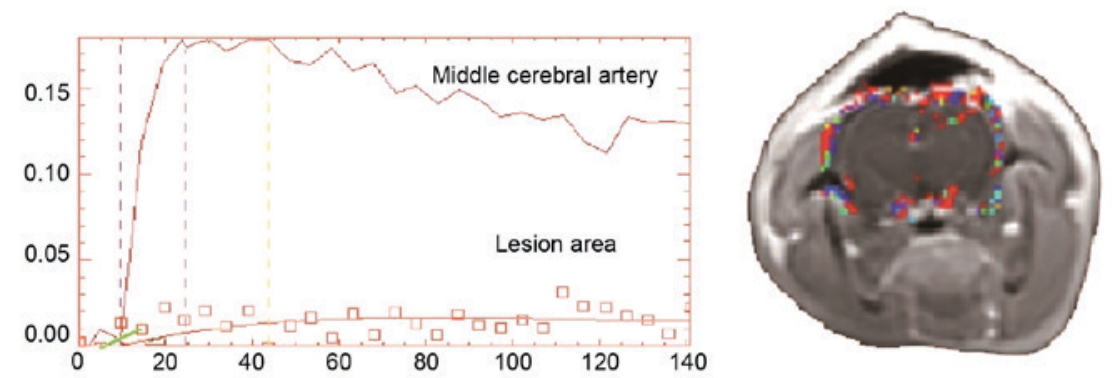

C
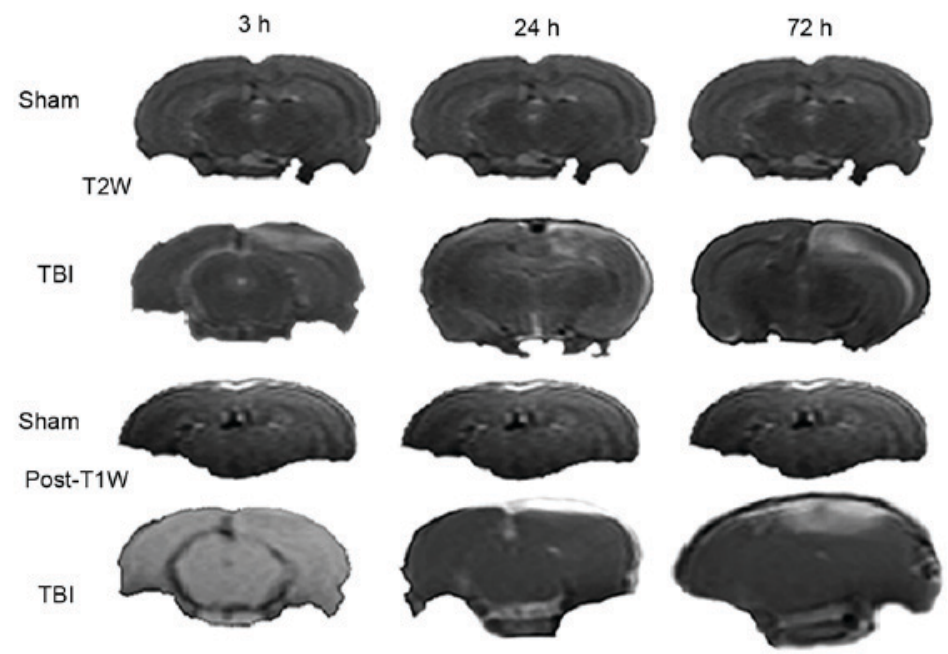

Figure 1. Representative MRI scans from a rat following the induction of TBI. Rats in the TBI group received a controlled cortical injury, whereas rats in the sham group were operated but received no brain insult. (A) Quantitative analysis of representative dynamic contrast-enhanced-MRI results at $72 \mathrm{~h}$ following the induction of TBI. The lower line represents the lesion area and the upper line indicates the middle cerebral artery. (B) $\mathrm{K}^{\text {trans }}$ map of the same rat. Blood brain barrier permeability was significantly increased at the site of the lesion. (C) T2W images demonstrate the area of lesion, whereas post contrast T1W images demonstrate gadolinium-diethylenetriaminepentacetate enhancement in the area of lesion at various time points post-TBI. MRI, magnetic resonance imaging; TBI, traumatic brain injury; $K^{\text {trans }}$, volume transfer constant; W, weighted.

(5.78 \pm 0.08 vs. $2.12 \pm 0.13 \mathrm{U} / \mathrm{mg}, \mathrm{P}<0.05)$ and $72 \mathrm{~h}(4.48 \pm 0.08$ vs. $2.12 \pm 0.24 \mathrm{U} / \mathrm{mg}, \mathrm{P}<0.05)$ following TBI compared with the sham group (Fig. 3C). Similarly, the decrease in T-AOC was also identified to be time-dependent within rats in the TBI group ( $\mathrm{P}<0.05$; Fig. 3C).

20-HETE production. LC-MS analysis demonstrated that the levels of 20-HETE were significantly upregulated in brain samples isolated from the area of injury at $3(4.26 \pm 0.17 \mathrm{vs}$. $3.08 \pm 0.18 \mathrm{ng} / \mathrm{mg}, \mathrm{P}<0.05), 24$ ( $7.74 \pm 0.11$ vs. $3.08 \pm 0.13 \mathrm{ng} / \mathrm{mg}$, $\mathrm{P}<0.05)$ and $72 \mathrm{~h}(9.82 \pm 0.08$ vs. $3.14 \pm 0.12 \mathrm{ng} / \mathrm{mg}, \mathrm{P}<0.05)$ post-TBI compared with the sham group (Fig. 3D). Furthermore, the increase in 20-HETE production was also identified to be time-dependent within rats in the TBI group $(\mathrm{P}<0.05$; Fig. 3D). The increase of 20-HETE suggests that the increase of the metabolite of AA following TBI.

Expression of JNK, c-Jun, ZO-1, occludin and MMP-9. The protein expression levels of JNK, c-Jun, ZO-1, occludin and MMP-9 in brain tissue samples isolated from the area of injury were assessed using western blot analysis. The present results demonstrated that the protein expression levels of p-JNK and p-c-Jun were significantly increased following the induction of TBI compared with the sham group $(\mathrm{P}<0.05)$, and the increase appeared to be time-dependent (Fig. 4A-D). Conversely, the expression of the tight junction proteins ZO-1 and occludin was observed to be significantly downregulated in TBI rats compared with that of rats from the sham group $(\mathrm{P}<0.05)$. Similarly, the decrease in ZO-1 and occludin protein expression levels appeared to be time-dependent (Fig. 4E-H). As presented in Fig. $4 \mathrm{I}$ and $\mathrm{J}$, the protein expression levels of MMP-9 were significantly upregulated, in a time-dependent manner, following the induction of TBI compared with the sham group $(\mathrm{P}<0.05)$.

\section{Discussion}

In the present study, brain water content was observed to be increased as early as $3 \mathrm{~h}$ following the induction of TBI, in accordance with previous reports demonstrating the development of brain edema $3 \mathrm{~h}$ following the onset of injury $(8,16)$. Notably, the present study demonstrated that 20-HETE levels were significantly increased 3,24 and $72 \mathrm{~h}$ following the induction of TBI compared with the sham group, thus suggesting that 20-HETE may contribute to the post-traumatic development of brain edema. These results suggested that 20-HETE may compromise 
A

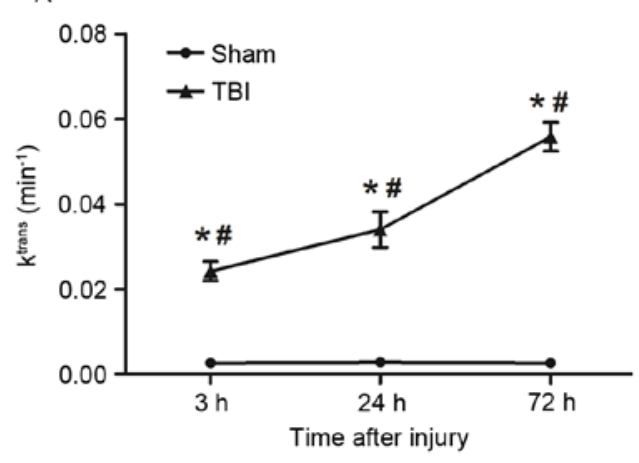

B

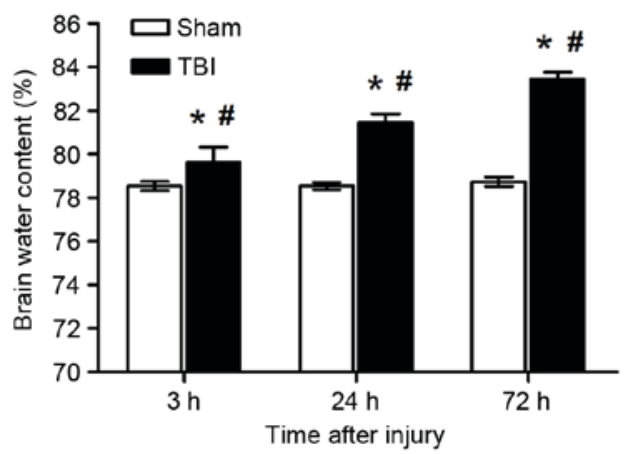

Figure 2. Blood brain barrier integrity was compromised in TBI rats, followed by the development of brain edema. Rats in the TBI group received a controlled cortical injury, whereas rats in the sham group were operated but received no brain insult. (A) $\mathrm{K}^{\text {trans }}$ values were calculated using dynamic contrast-enhanced-magnetic resonance imaging. $\mathrm{K}^{\text {trans }}$ values were higher in the lesion area following the induction of TBI compared with the sham group at all time points. (B) Brain water contents were assessed using the wet/dry ratio method. Water contents were significantly increased in the traumatic brain. Data are expressed as the mean \pm standard deviation. ${ }^{*} \mathrm{P}<0.05$ vs. the sham group at the same time point; ${ }^{\sharp} \mathrm{P}<0.05$ vs. the TBI group at the adjacent time point. TBI, traumatic brain injury; $\mathrm{K}^{\text {trans }}$, volume transfer constant.

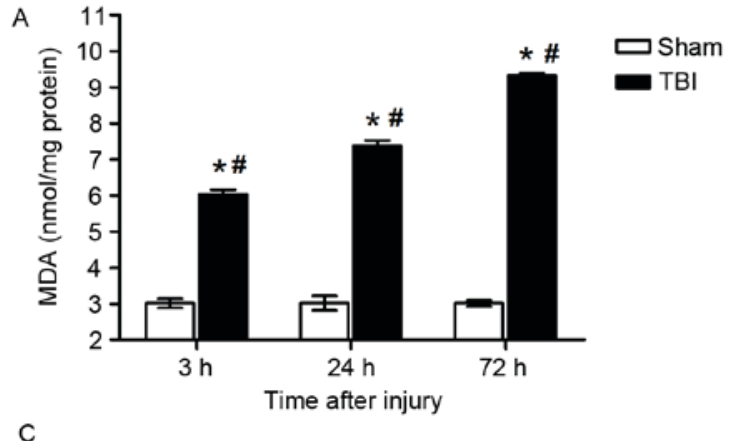

C

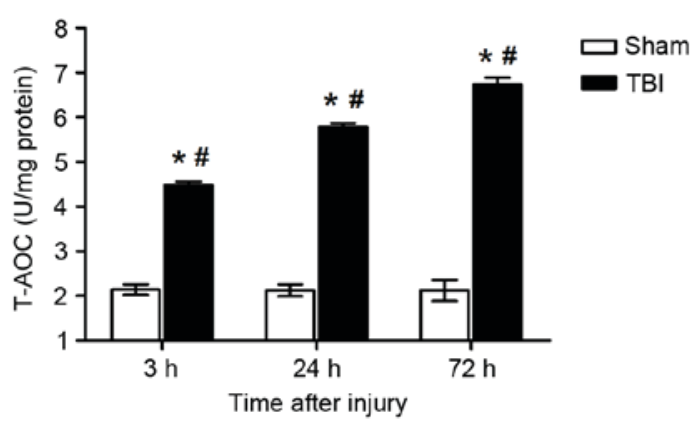

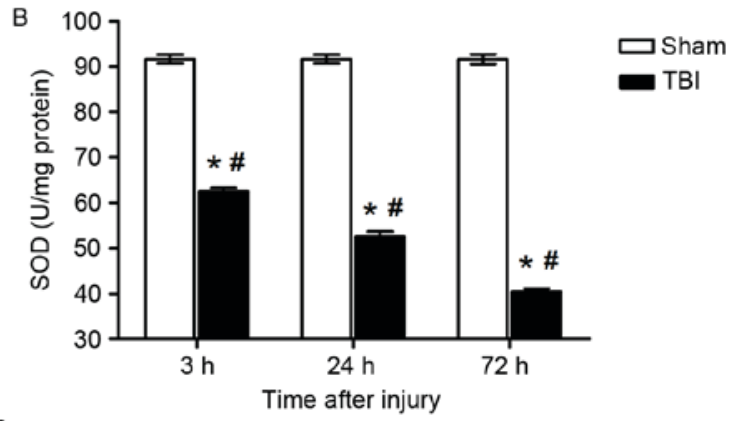

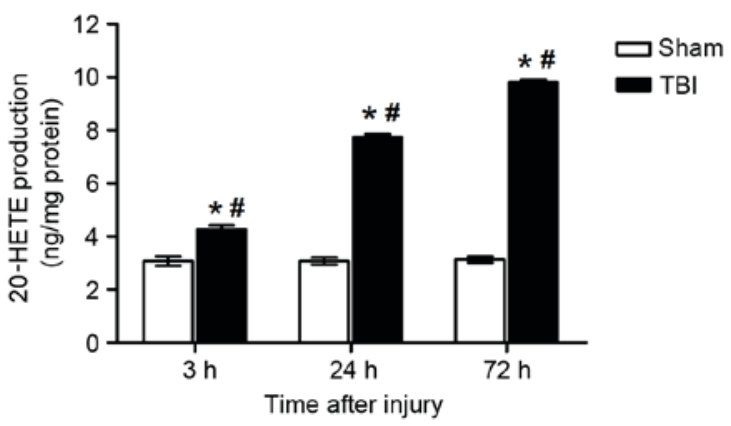

Figure 3. MDA and 20-HETE levels, SOD activity and T-AOC were assessed following the induction of TBI. Rats in the TBI group received a controlled cortical injury, whereas rats in the sham group were operated but received no brain insult. (A) MDA contents in the area of lesion were significantly increased whereas (B) the activity of SOD and (C) T-AOC were decreased following TBI. (D) 20-HETE production was significantly increased in traumatic brain tissue. Data are expressed as the mean \pm standard deviation. ${ }^{*} \mathrm{P}<0.05$ vs. the sham group at the same time point; ${ }^{*} \mathrm{P}<0.05$ vs. the TBI group at the adjacent time point. MDA, malondialdehyde; HETE, hydroxyeicosatetraenoic acid; SOD, superoxide dismutase; T-AOC, total antioxidative capacity; TBI, traumatic brain injury.

the integrity and enhance the permeability of the BBB, thus participating in the development of brain edema following TBI.

Traumatic brain edema has been reported to consist of cytotoxic and vasogenic edema (8). Cytotoxic edema has been suggested to occur within 30 min following the brain insult, and has been attributed to the activation of inflammatory processes, the increase in oxidative stress and the enzymatic degradation of the extracellular matrix in the BBB $(16,17)$. Conversely, vasogenic edema has been reported to appear several $\mathrm{h}$ following the onset of TBI $(16,17)$. The results of the present study suggested that 20-HETE may influence the brain water contents during the initial $3 \mathrm{~h}$ following injury, probably through the potentiation of pathways contributing to the development of cytotoxic edema at the early post-injury phase.

To further investigate the molecular mechanisms underlying the implication of 20-HETE in the development of brain edema, ROS generation was assessed post-TBI. The present results demonstrated that ROS production was significantly increased following the induction of TBI compared with the sham group, and suggested that the aberrant ROS generation may contribute to the dysregulation of the BBB. Furthermore, these observations suggested that the BBB-compromising effects of 20-HETE during the development of brain edema may be mediated by an increase in oxidative stress. 
A

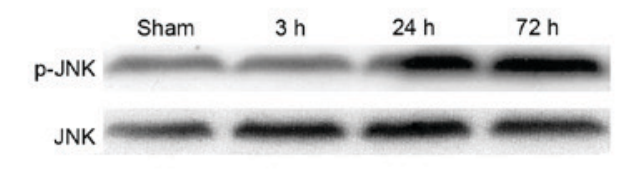

c

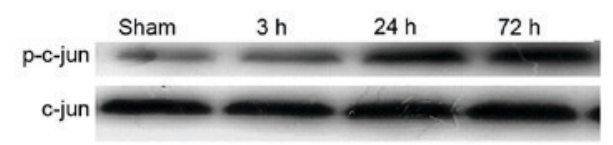

E

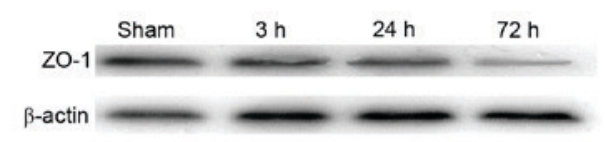

G

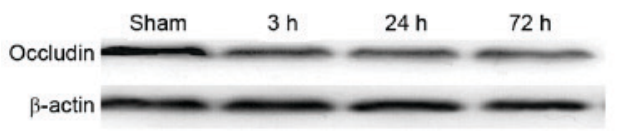

1

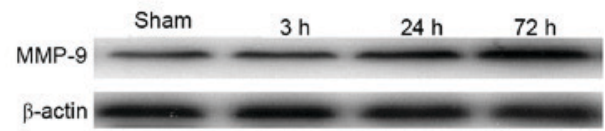

B
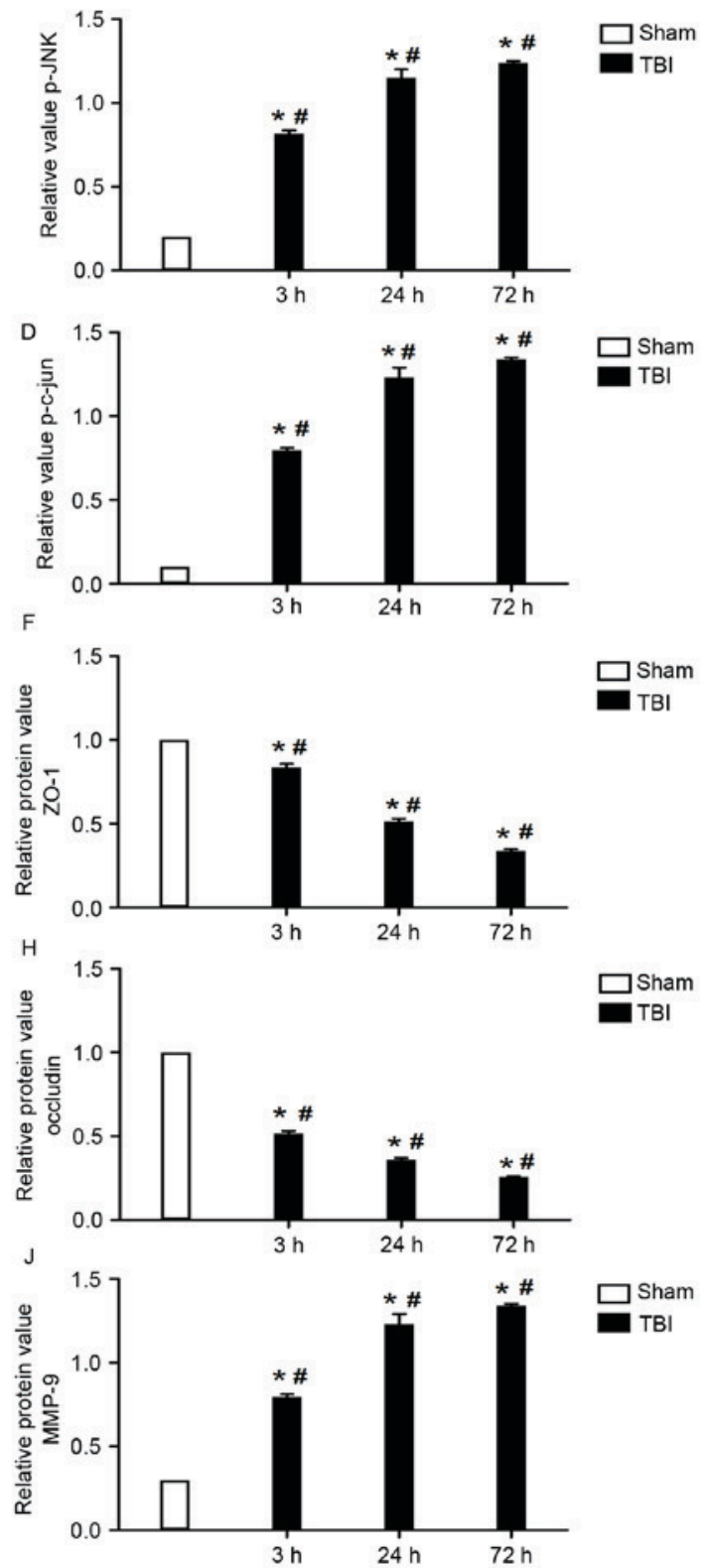

Figure 4. Western blot analysis was used to assess the expression levels of the JNK pathway proteins p-JNK and p-c-Jun, of the tight junction proteins ZO-1 and occludin, and of MMP-9 following the induction of TBI. Rats in the TBI group received a controlled cortical injury, whereas rats in the sham group were operated but received no brain insult. The protein expression levels of (A and B) p-JNK, (C and D) p-c-Jun and (I and J) MMP-9 were significantly upregulated in the area of lesion. The protein expression levels of (E and F) ZO-1 and $(\mathrm{G}$ and $\mathrm{H})$ occludin were significantly downregulated in the area of lesion. Data are expressed as the mean \pm standard deviation. ${ }^{*} \mathrm{P}<0.05$ vs. the sham group; ${ }^{~} \mathrm{P}<0.05$ vs. the TBI group at the lower time point. JNK, c-Jun $\mathrm{N}$-terminal kinase; $\mathrm{p}-$, phosphorylated; ZO, zonula occludens; MMP, matrix metalloproteinase; TBI, traumatic brain injury.

Previous studies have suggested a strong association between oxidative stress and MMP-9 in the pathophysiology of BBB damage following TBI $(18,19)$, as oxidative stress has been reported to trigger molecular cascades that mediate MMP-9 activation (20). MMP-9 activation can lead to the degradation of tight junction proteins, such as occludin and ZO-1, and thus increase BBB permeability following injury (21). The present study identified that the expression of MMP-9 was enhanced following the induction of TBI, in accordance with previous reports $(20,21)$. In addition, the expression of the tight junction proteins occludin and ZO-1 was significantly downregulated following TBI. These results suggested that 20-HETE may modulate the expression of MMP-9 and subsequently of BBB tight junction proteins, and thus contribute to the development of brain edema post-TBI.

The JNK signaling pathway has been implicated in neuronal injury triggered by TBI-induced increases in oxidative stress $(22,23)$, whereas the inhibition of JNK has been identified to prevent BBB disruptions, via inhibiting the activation of MMP-9 (15). These observations suggested that the JNK pathway may serve an important role in the regulation of tight junction proteins and the maintenance of BBB integrity. The present study demonstrated that the activation of JNK and its downstream transcription factor c-Jun was enhanced following TBI, thus suggesting that 20-HETE 
may regulate MMP-9 expression through the modulation of the JNK intracellular signaling pathway. Yu et al (24) reported that 20-HETE upregulated the expression of MMP-9 through phosphatidylinositol-4,5-bisphosphate 3-kinase and extracellular signal-regulated kinase-mediated signaling pathways in human non-small cell lung cancer cells, thus suggesting an association between 20-HETE and MMP-9. However, the molecular mechanisms underlying the regulatory effects of 20-HETE on MMP-9 expression may differ across species and cell types. The results of the current study suggested that increased ROS generation may be implicated in the mechanisms underlying the effects of 20-HETE on MMP-9; however, Yu et al (24) reported that the effects of 20-HETE on MMP-9 appeared to be independent of ROS. Fordsmann et al (25) demonstrated that the 20-HETE inhibitor HET0016 ameliorated the reduction in cerebral blood flow in a rat brain injury model. Therefore, the mechanisms underlying the 20-HETE-mediated BBB impairment and brain edema development may also involve the disruption of cerebral blood flow.

In conclusion, the present results suggested that 20-HETE may be involved in the compromise of BBB integrity and the development of brain edema following TBI. In addition, the present study suggested that the molecular mechanisms underlying the implication of 20-HETE on BBB dysfunction may involve an increase in oxidative stress and in the expression of MMP-9, thus contributing to the degradation of tight junction proteins, possibly through the activation of the JNK signaling pathway. Thus, based on this study, the inhibition of 20-HETE may be used in future experiments and another mechanism and treatment choice may be explored in clinical experiments.

\section{Acknowledgements}

The present study was supported by the National Natural Science Foundation of China (grant nos. 81271540 and 81301213).

\section{References}

1. Birnie M, Morrison R, Camara R and Strauss KI: Temporal changes of cytochrome P450 (Cyp) and eicosanoid-related gene expression in the rat brain after traumatic brain injury. BMC Genomics 14: 303, 2013.

2. Yang S, Ma Y, Liu Y, Que H, Zhu C and Liu S: Arachidonic acid: A bridge between traumatic brain injury and fracture healing. J Neurotrauma 29: 2696-2705, 2012.

3. Johnson AL, Edson KZ, Totah RA and Rettie AE: Cytochrome P450 $\omega$-hydroxylases in inflammation and cancer. Adv Pharmacol 74: 223-262, 2015.

4. Harder DR, Gebremedhin D, Narayanan J, Jefcoat C, Falck JR, Campbell WB and Roman R: Formation and action of a P-450 $4 \mathrm{~A}$ metabolite of arachidonic acid in cat cerebral microvessels. Am J Physiol 266: H2098-H2107, 1994.

5. Zhu J, Wang B, Lee JH, Armstrong JS, Kulikowicz E, Bhalala US, Martin LJ, Koehler RC and Yang ZJ: Additive neuroprotection of a 20-HETE inhibitor with delayed therapeutic hypothermia after hypoxia-ischemia in neonatal piglets. Dev Neurosci 37: 376-389, 2015.

6. Garcia V, Cheng J, Weidenhammer A, Ding Y, Wu CC, Zhang F, Gotlinger K, Falck JR and Schwartzman ML: Androgen-induced hypertension in angiotensinogen deficient mice: Role of 20-HETE and EETS. Prostaglandins Other Lipid Mediat 116-117: 124-130, 2015.
7. Toth P, Csiszar A, Sosnowska D, Tucsek Z, Cseplo P, Springo Z, Tarantini S, Sonntag WE, Ungvari Z and Koller A: Treatment with the cytochrome P450 $\omega$-hydroxylase inhibitor HET0016 attenuates cerebrovascular inflammation, oxidative stress and improves vasomotor function in spontaneously hypertensive rats. Br J Pharmacol 168: 1878-1888, 2013.

8. Wei XE, Zhang YZ, Li YH, Li MH and Li WB: Dynamics of rabbit brain edema in focal lesion and perilesion area after traumatic brain injury: A MRI study. J Neurotrauma 29: 2413-2420, 2012.

9. Cai H, Mu Z, Jiang Z, Wang Y, Yang GY and Zhang Z: Hypoxia-controlled matrix metalloproteinase- 9 hyperexpression promotes behavioral recovery after ischemia. Neurosci Bull 31: 550-560, 2015.

10. Xu FF, Sun S, Ho AS, Lee D, Kiang KM, Zhang XQ, Wang AM, Wu EX, Lui WM, Liu BY and Leung GK: Effects of progesterone vs. dexamethasone on brain oedema and inflammatory responses following experimental brain resection. Brain Inj 28: 1594-1601, 2014.

11. Kim JY, Ko AR, Hyun HW and Kang TC: ETB receptor-mediated MMP-9 activation induces vasogenic edema via $\mathrm{ZO}-1$ protein degradation following status epilepticus. Neuroscience 304: 355-367, 2015.

12. Wu G, Wu J, Jiao Y, Wang L, Wang F and Zhang Y: Rosiglitazone infusion therapy following minimally invasive surgery for intracerebral hemorrhage evacuation decreases matrix metalloproteinase-9 and blood-brain barrier disruption in rabbits. BMC Neurol 15: 37, 2015.

13. Wang GY, Wang N and Liao HN: Effects of muscone on the expression of P-gp, MMP-9 on blood-brain barrier model in vitro. Cell Mol Neurobiol 35: 1105-1115, 2015

14. Liu Y, Wang D, Wang H, Qu Y, Xiao X and Zhu Y: The protective effect of HET0016 on brain edema and blood-brain barrier dysfunction after cerebral ischemia/reperfusion. Brain Res 1544: 45-53, 2014.

15. Urrutia A, Rubio-Araiz A, Gutierrez-Lopez MD, ElAli A, Hermann DM, O'Shea E and Colado MI: A study on the effect of JNK inhibitor, SP600125, on the disruption of blood-brain barrier induced by methamphetamine. Neurobiol Dis 50: 49-58, 2013.

16. Jungner M, Siemund R, Venturoli D, Reinstrup P, SCHALéN W and Bentzer P: Blood-brain barrier permeability following traumatic brain injury. Minerva Anestesiol 82: 525-533, 2016.

17. Hanrahan F and Campbell M (eds.): Frontiers in neuroscience neuroinflammation. In: Translational research in traumatic brain injury. Laskowitz D and Grant G (eds.) CRC Press/Taylor and Francis Group (c) 2016 by Taylor \& Francis Group LLC. Boca Raton (FL), 2016.

18. Gasche Y, Copin JC, Sugawara T, Fujimura M and Chan PH: Matrix metalloproteinase inhibition prevents oxidative stress-associated blood-brain barrier disruption after transient focal cerebral ischemia. J Cereb Blood Flow Metab 21: 1393-1400, 2001.

19. Lapchak PA, Chapman DF and Zivin JA: Metalloproteinase inhibition reduces thrombolytic (tissue plasminogen activator)-induced hemorrhage after thromboembolic stroke. Stroke 31: 3034-3040, 2000.

20. Gu JH, Ge JB, Li M, Xu HD, Wu F and Qin ZH: Poloxamer 188 protects neurons against ischemia/reperfusion injury through preserving integrity of cell membranes and blood brain barrier. PLoS One 8: e61641,2013.

21. Wang ZG, Cheng Y, Yu XC, Ye LB, Xia QH, Johnson NR, Wei X, Chen DQ, Cao G, Fu XB, et al: bFGF protects against blood-brain barrier damage through junction protein regulation via PI3K-Akt-Racl pathway following traumatic brain injury. Mol Neurobiol 53: 7298-7311, 2015.

22. Liu Y, Wang H, Zhu Y, Chen L, Qu Y and Zhu Y: The protective effect of nordihydroguaiaretic acid on cerebral ischemia/reperfusion injury is mediated by the JNK pathway. Brain Res 1445: 73-81, 2012.

23. Mehta SL, Manhas N and Raghubir R: Molecular targets in cerebral ischemia for developing novel therapeutics. Brain Res Rev 54: 34-66, 2007.

24. Yu W, Chen L, Yang YQ, Falck JR, Guo AM, Li Y and Yang J: Cytochrome P450 w-hydroxylase promotes angiogenesis and metastasis by upregulation of VEGF and MMP-9 in non-small cell lung cancer. Cancer Chemother Pharmacol 68: 619-629, 2011.

25. Fordsmann JC, Ko RW, Choi HB, Thomsen K, Witgen BM, Mathiesen C, Lønstrup M, Piilgaard H, MacVicar BA and Lauritzen M: Increased 20-HETE synthesis explains reduced cerebral blood flow but not impaired neurovascular coupling after cortical spreading depression in rat cerebral cortex. J Neurosci 33: 2562-2570, 2013. 